\title{
Continuous Monitoring of Patient Mobility for 18 Months Using Inertial Sensors following Traumatic Knee Injury: A Case Study
}

\author{
Arne Mueller $^{a} \quad$ Holger Hoefling $^{b} \quad$ Timur Nuritdinow $^{c} \quad$ Nicholas Holway $^{b}$ \\ Matthias Schieker ${ }^{a}$ Martin Daumer ${ }^{d}$ leuan Clay ${ }^{a}$ \\ aTranslational Medicine, Novartis Institutes for Biomedical Research, Forum 1, Novartis \\ Campus, Basel, Switzerland; b NIBR Informatics, Novartis Institutes for Biomedical Research, \\ Forum 1, Novartis Campus, Basel, Switzerland; ' Sylvia Lawry Centre eV, The Human Motion \\ Institute, Munich, Germany; ${ }^{d}$ TRIUM Online Analysis GmbH, Munich, Germany
}

\section{Keywords}

Activity monitors · Algorithms · Data · Mobility · Wearable physical activity monitoring ·

Wearable sensors

\section{Abstract}

Continuous patient activity monitoring during rehabilitation, enabled by digital technologies, will allow the objective capture of real-world mobility and aligning treatment to each individual's recovery trajectory in real time. To explore the feasibility and added value of such approaches, we present a case study of a 36-year-old male participant monitored continuously for activity levels and gait parameters using a waist-worn inertial sensor following a tibial plateau fracture on the right side, sustained as a result of a high-energy trauma during a sporting accident. During rehabilitation, data were collected for a period of 553 days, with $>80 \%$ daytime compliance, until the participant returned to near full mobility. The participant completed a daily diary with the annotation of major events (falls, near falls, cycling periods, or physiotherapy sessions) and key dates in the patient's recovery, including medical interventions, transitioning off crutches, and returning to work. We demonstrate the feasibility of collecting, storing, and mining of continuous digital mobility data and show that such data can detect changes in mobility and provide insights into long-term rehabilitation. We make both raw data and annotations available as a resource with the aspiration that further methods and insights will be built on this initial exploration of added value and continue to demonstrate that continuous monitoring can be deployed to aid rehabilitation. 
Mueller et al.: Continuous Monitoring of Patient Mobility for 18 Months Using Inertial Sensors following Traumatic Knee Injury: A Case Study

\section{Introduction}

Assessment of mobility within a controlled or supervised environment [1] allows patient therapy to be tailored, aided in large part by our increasing ability to measure a patient's performance using a combination of prescribed movement and sensor analytics [2, 3]. However, such snapshot assessments tend to have high inter-test variability, driven by patient motivation, short-term changes in fatigue, and other factors [4].

Recent research has shown that unsupervised, sensor-based monitoring of rehabilitation exercise in the home environment can both improve adherence and enable objective scoring as a basis for feedback [5], providing patients are sufficiently motivated [6]. However, improved performance assessment results and ability to perform rehabilitation exercises only partially capture the recovery process, and the same technologies could also be used to monitor long-term, real-world mobility behaviors. Understanding the relationship between performance changes, their consequence on behavioral patterns and ultimately subjective perceptions of independence and quality of life will enable the examination of the ecological validity of performance testing and hopefully a more complete chain of evidence demonstrating the efficacy of new therapeutic interventions. This case study describes how continuous monitoring of a participant's mobility could be used to track overall trends and specific events in the participant's rehabilitation following a severe knee injury.

\section{Case Report}

\section{Accelerometry}

The patient was continuously monitored using a waist-worn inertial sensor (TRIUM actibelt RCT2, [7]), which recorded acceleration in 3-dimensions at a sampling frequency of $100 \mathrm{~Hz}$ (12-bit resolution) and a range of $\pm 6 \mathrm{~g}$. Raw acceleration data was collected for a period of 553 days, from postinjury day 15 and post discharge day 6 following initial surgery, until the participant returned to near full mobility. Multiple devices, each of the same model, design, and placement were used over the observation period. Each individual device was worn continuously for a period of up to 75 days (mean 33 days) without requiring charging or other interaction and were otherwise self-managed by the participant. Recorded data was stored locally on the device and was downloaded periodically. Resulting recordings were then merged to form a single raw data file, which is made available as a set of text files.

\section{Patient Diary}

The patient completed a daily diary annotating periods of immobility, crutch-assisted walking, cycling, as well as unassisted walking. All major events, including falls, near falls, appointments with his doctors, and physiotherapy sessions were captured. The diaries are available as text files.

\section{Derived Annotations}

Further algorithmically derived annotations for bouts of walking were also included. Steps were detected by combining Continuous Wavelet Transformation-derived temporal gait features (adapted from [8]). Steps were then annotated with the estimated gait speed peak using supervised methods $[9,10]$ and were further classified as left and right foot steps using lateral acceleration patterns. We defined "bouts" as series of contiguous steps with no 2 steps separated by more than an empirically defined threshold of $3 \mathrm{~s}$. Step detection may produce false-positive steps for periodic non-walking behavior such as cycling, thus known cycling periods were removed from our analysis. 
Mueller et al.: Continuous Monitoring of Patient Mobility for 18 Months Using Inertial Sensors following Traumatic Knee Injury: A Case Study

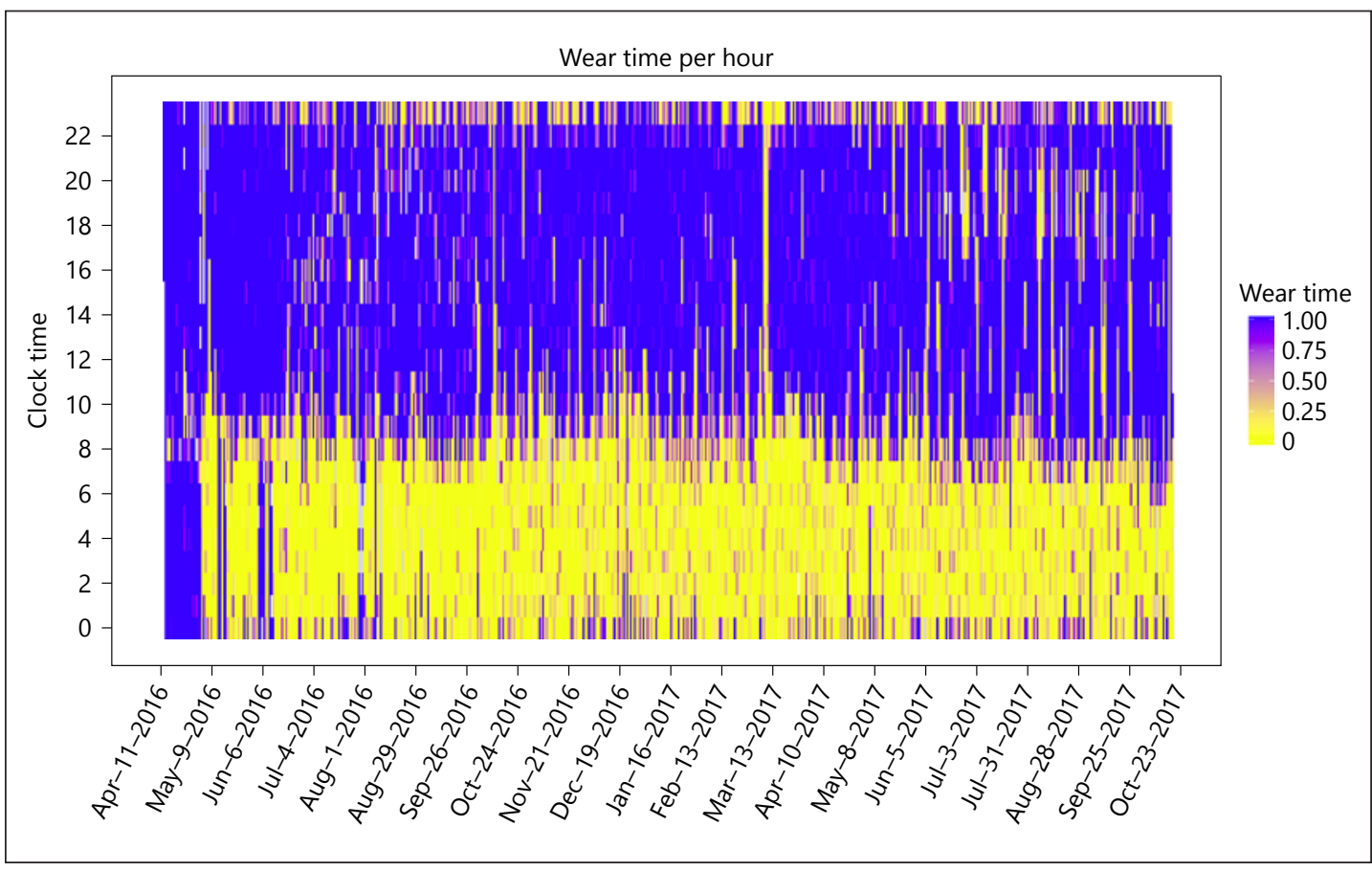

Fig. 1. Hourly wear time for the entire data collection period. The horizontal axis represents the date during the observation period, and the vertical axis represents a 24-h clock, with each vertical line therefore representing a given day. Wear time was summarized for each hour of each day and was projected to a color gradient: yellow indicates no or a low hourly wear time and deep blue a high or a full wear time (1.0 corresponds to $60 \mathrm{~min}$ of wear time within $1 \mathrm{~h}$ ). Wear time varied by the time of the day, generally being higher during daytime hours as compared to the night.

\section{How to Access}

Written informed consent was obtained from the participant for the collection and use of the data, and a local ethics committee waiver was granted for the collection and publication of the data sets, which can be accessed via https://github.com/Novartis.

\section{Results}

\section{Participant Compliance}

Treatment and rehabilitation for severe acute musculoskeletal injuries or chronic mobility disorders can take many years $[11,12]$. It is therefore important to demonstrate that high density, high compliance monitoring in an uncontrolled environment is possible over such a time. The participant collected raw 3D accelerometry data over a period of 553 days at a mean compliance of $61 \%$ for the total monitoring period, and $84 \%$ during daytime hours (08:00 to $22: 59)$. In total, $13,265 \mathrm{~h}$ of raw accelerometry data were collected during the observation period ( $\sim 14.3$ billion data points, $>3.6$ million detected steps). Figure 1 shows the compliance over the full observation period.

\section{Changes in Standard Mobility Parameters during Rehabilitation}

Rehabilitation from this type of knee injury focusses on regaining joint flexibility and muscle strength to allow the patient to regain normal mobility [13]. A key component of mobility is walking, typically measured in terms of step counts and gait speed. We developed 
Mueller et al.: Continuous Monitoring of Patient Mobility for 18 Months Using Inertial

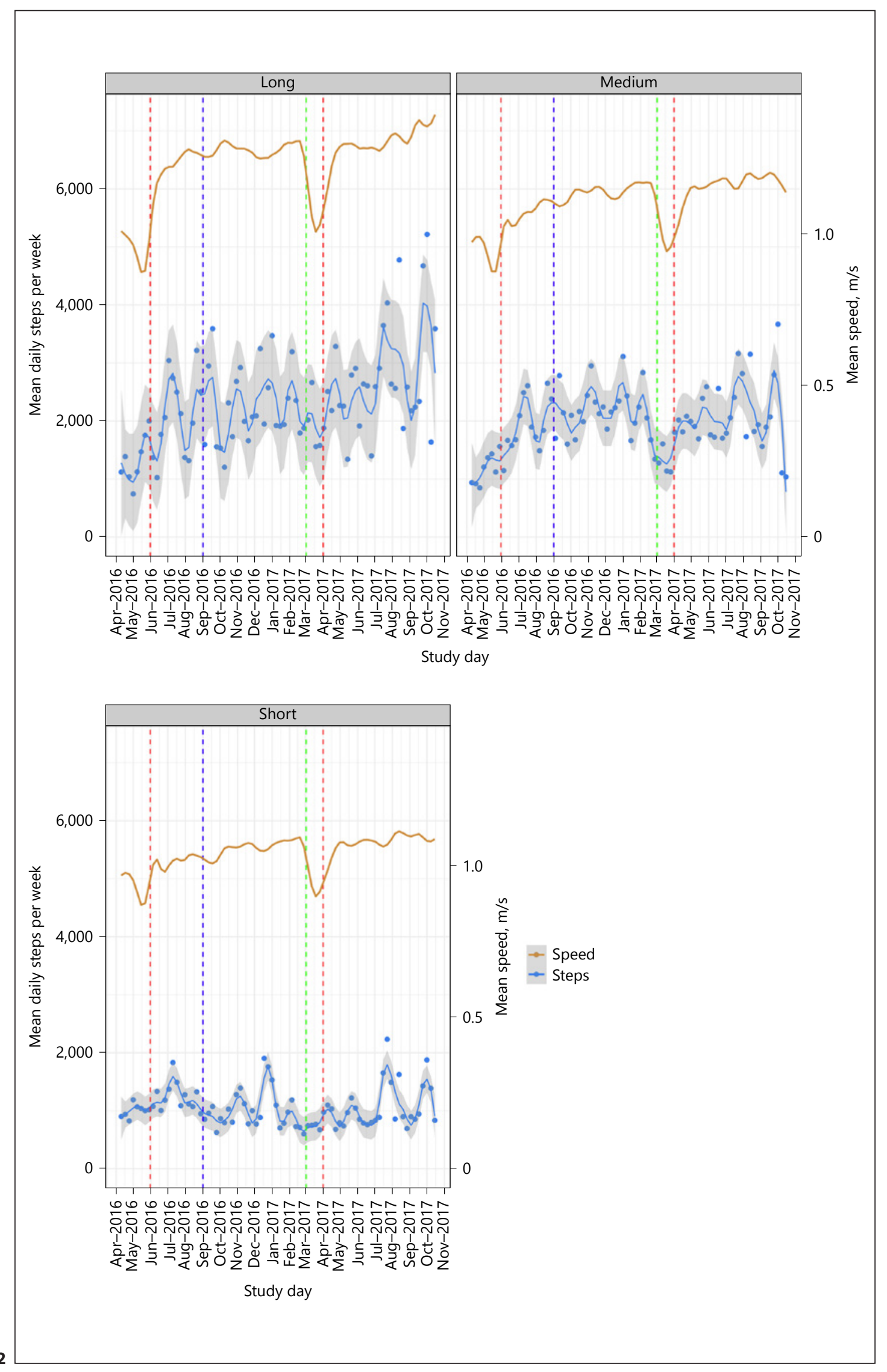


Mueller et al.: Continuous Monitoring of Patient Mobility for 18 Months Using Inertial Sensors following Traumatic Knee Injury: A Case Study

a step detection algorithm based on continuous wavelet transformation [8] and for each step estimated gait speed using a support vector regression model [10]. We observed that walking behavior differs strongly between bouts of different length, for example the average gait speed in longer bouts tends to be higher.

To allow fairer comparisons over time, comparing similar bouts rather than general walking behavior, which may change markedly over the course of rehabilitation, we divided bouts into groups of 3 duration ranges. Figure 2 shows 3 panels with the mean daily step count and mean gait speed plotted against time, annotated with key dates in the participant's rehabilitation. Each panel groups long, medium, and short bouts of walking; 101 to 500, between 21 and 100, and between 5 and 20 contiguous steps, respectively.

We observed a sudden and rapid increase in the number of daily steps during the first 4 months after surgery for long and medium bouts but less so for short bouts. The data also shows an immediate reduction in step counts, in particular for long bouts, after the participant returned to full-time work (blue vertical dashed line, Fig. 2). We also observe a shortterm decrease in the number of steps and gait speed following the second surgery and the participant no longer requiring crutches. The variation in average daily steps per week was larger in longer bouts than for medium and short bouts, probably due to the larger range of steps for longer bouts ( $>100-500$ steps) than for medium ( $>20-100$ steps) bouts.

This suggests that it is possible to detect overall mobility recovery using our approach. The data also highlights some of the challenges in interpreting such data, for example the noticeable reduction in mobility upon returning to work. This is an interesting observation as typically any reduction in mobility would be interpreted as a negative outcome, yet in this case it is an indication of a positive transition in the participant's rehabilitation, i.e., returning to work at their office-based job. Moreover, large absolute changes in step count or gait speed, or bout length distributions (see online suppl. Fig. 1; for all online suppl. material, see www. karger.com/doi/10.1159/000490919) were only observed immediately following the initial injury and therefore do not fully describe the progression in the rehabilitation of the participant from near immobility to returning to near normal daily mobility.

\section{Changes in Gait Characteristics during Rehabilitation}

We additionally sought to characterize the participant's recovery by examining gait asymmetry. The participant suffered a unilateral injury on the right side, so we hypothesized that improving weight-bearing ability would be concomitant with a return to a more symmetric gait $[14,15]$. We used lateral acceleration patterns to annotate detected steps as left or right footed. Figure 3 shows the mean daily stance time (time between heel strike and toe-off events, i.e., the amount of time the foot spends on the ground) for left and right foot steps for the full observation period. As for Figure 1, data is again grouped into long, medium, and short bouts and annotated with major events. We observe an overall reduction in stance times on both sides during rehabilitation, which match increased gait speeds as shown in Figure 2, and

Fig. 2. Gait speed and the average step count over the entire observation period. Each panel shows, on the vertical axis, the average daily number of steps per week (blue dots and blue smoothed line [Loess] with the scale on the left-hand vertical axis) and the mean weekly gait speed (brown smoothed line [Loess] and the scale on the right-hand axis) over the full observation period (horizontal axis). The vertical red, blue, green, and red dashed lines indicate the following events: first time off crutches, return to work, 2nd surgery, and off crutches. Each panel represents bouts of continuous walking without interruption with 5-20 steps (short), 21-100 (medium) steps, and 101-500 steps, right to left, respectively. Distributions and patterns of gait speed and the average step counts differ strongly between the different bout lengths (different panels). Large increases in step counts and gait speed were only seen immediately following the initial injury. 
Mueller et al.: Continuous Monitoring of Patient Mobility for 18 Months Using Inertial Sensors following Traumatic Knee Injury: A Case Study

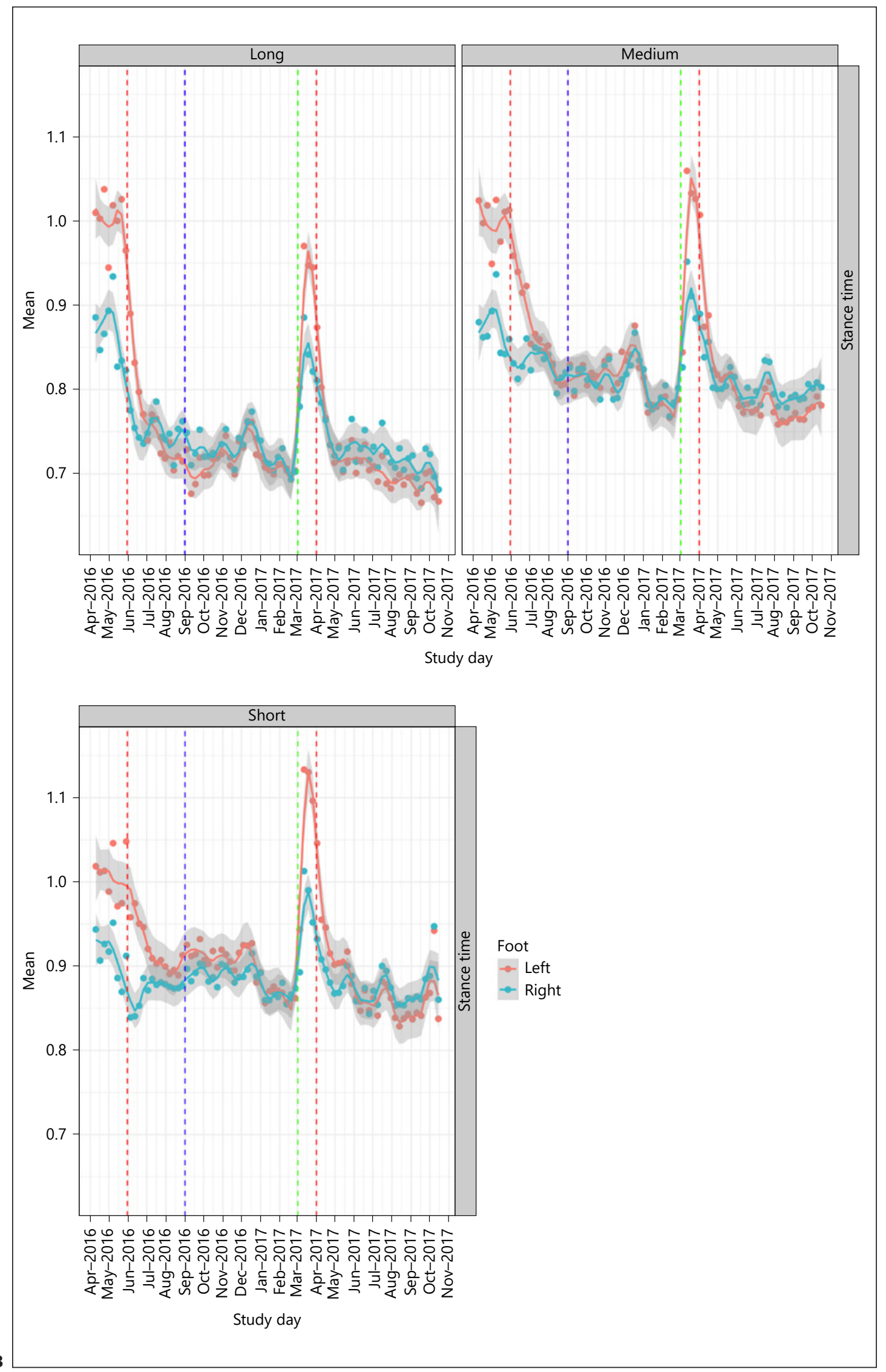


Mueller et al.: Continuous Monitoring of Patient Mobility for 18 Months Using Inertial Sensors following Traumatic Knee Injury: A Case Study

that stance times for short bouts are generally higher, again matching the generally slower gait speed.

Gait characteristics appear to better reflect the participants rehabilitation than the general walking parameters, as shown in Figure 2, as there is a clear difference in stance time for the left and right (affected) side at the beginning of the study, converging at around $0.7 \mathrm{~s}$ (in long bouts) for both sides. Recovery from the 2nd surgery was faster than after the 1st surgery, shown by the quicker convergence of stance times after the participant stopped using crutches for the second time, possibly due to complications, including deep vein thrombosis, following the first surgery.

These results show the value of capturing continuous raw accelerometry data, which enables deeper re-examination of recovery. In this case study, we demonstrate the feasibility of long-term digital monitoring of rehabilitation and the potential to derive insights meaningful to both patients and practitioners. We show that gait speed is affected during rehabilitation and that measures of gait symmetry further help describe major phases and transitions in recovery. It should be noted that a return to baseline gait characteristics (e.g., gait speed or gait symmetry) may not uncover all aspects of recovery; for example, a patient's gait may increase over time after injury, but with an altered unhealthy walking style. We hope that further work will enable a more complete understanding of parameters relevant to mobility.

\section{Minimum Required Compliance}

The high level of compliance seen in this case study may, however, not be possible or necessary in other settings, so we also investigated what minimal level of compliance would be required to observe the same trends. We simulated reduced compliance, or a less burdensome patient instruction, by down-sampling the data set to simulate shorter and sparser periods of monitoring. Figure 4 shows that for these parameters, a wear time of only 6 continuous days per month and only $6 \mathrm{~h}$ wear time per day are required to capture longterm trends and changes over time in weekly averages.

It is important to note that detailed information gets lost with more sparse compliance, for example, we are less able to accurately capture periods of high variation (e.g., immediately after surgery) or specific rare events (e.g., falls), and that any outliers that are captured will have a stronger influence on observed trends. Furthermore, we note that 6 continuous days of monitoring per month results in less variation in weekly average trends than 6 randomly sampled days per month, or more widely dispersed but larger blocks (e.g., 52 -month periods). As a consequence, researchers should carefully balance instructions given to patients with the needs of their study; if high variability (for example early in rehabilitation) or rare events are expected, then teams must ensure sites, and patients are sufficiently motivated and compliant to capture informative data. It is worth noting that the participant-provided feedback on their experience and their motivation was improved by sharing their data and results: "I liked having a report every month or so to track my recovery. As my recovery progressed there were fewer 'milestones' (first steps, return to sports etc.) compared to the early stages so it was motivating to see that I was continuing to improve."

Fig. 3. Stance time per foot over the entire data collection period. Each panel represents, on the vertical axis, the average stance time (time spent on 1 foot) per week while walking over the entire study duration (horizontal axis). The red data set is for the left foot and the blue-green data set for the right foot (affected side). See the legend of Figure 2 for the details on the annotation. Differences in stance time (interpreted as gait asymmetry) closely reflect major events (for example, the most extreme differences are seen around the first and second surgery) and overall trends in rehabilitation. 
Mueller et al.: Continuous Monitoring of Patient Mobility for 18 Months Using Inertial

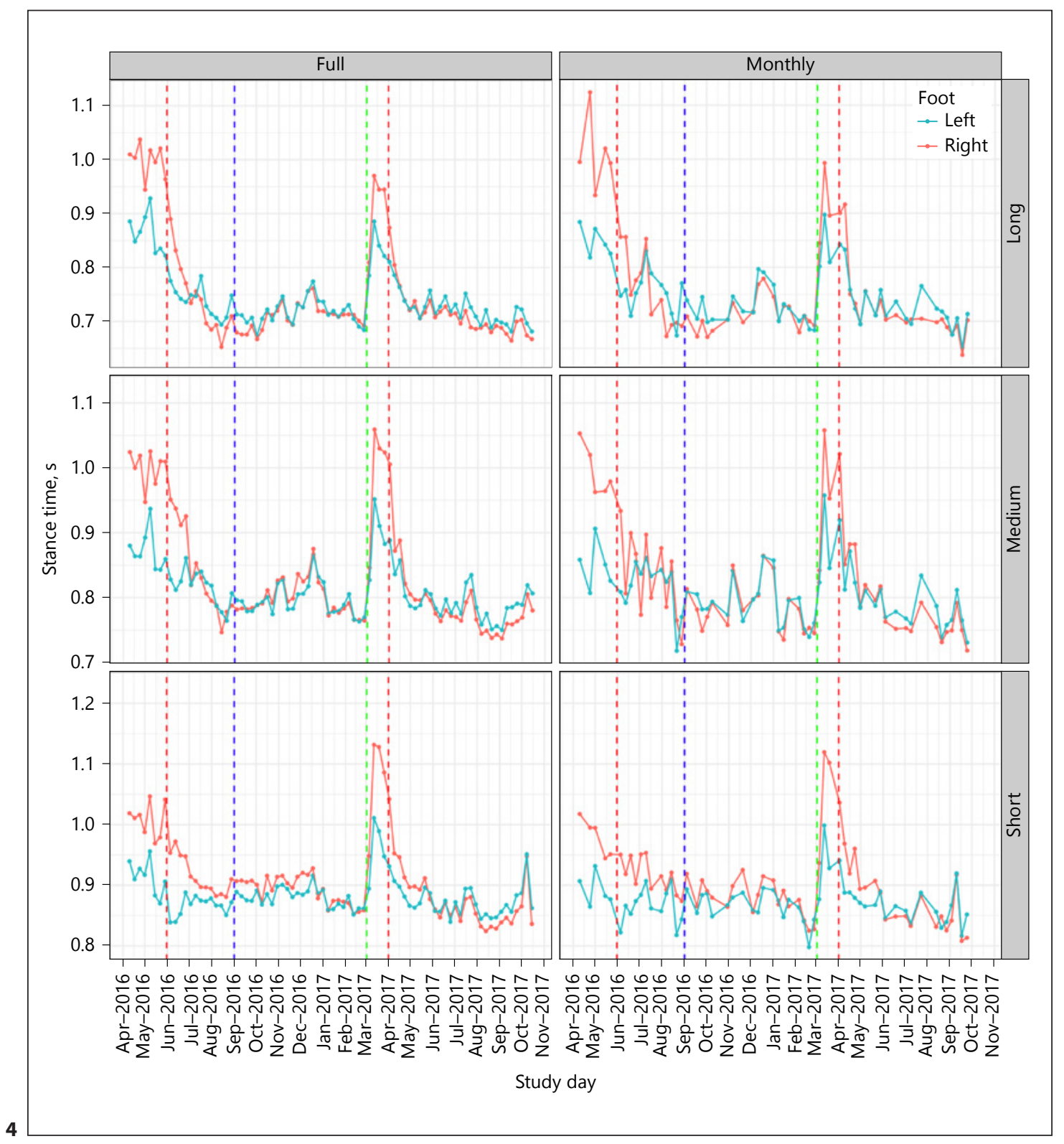

(Figure continued on next page.)

\section{Conclusions}

This case study demonstrates that long-term, continuous, real-world monitoring of mobility is feasible at high levels of compliance, and that data captured during this period describes major trends and events in the participant's rehabilitation. We show that classic measures of overall walking ability (number of steps and gait speed) are less informative in this setting than measurements of gait symmetry (stance time for each foot). We also demonstrate that such a high compliance is not required for capturing these major trends in rehabilitation but are, however, required to capture rare events such as falls.

While we do clearly see trends over time that correspond to major events in the participant's rehabilitation, it is important to note that the methods described here in 1 participant require careful and appropriately powered validation against gold-standard methods [9] to evaluate their accuracy, sensitivity, and ability to detect clinically meaningful changes and 
.... digital

DI@Tarkers

Fig. 4. Required wear time and participant compliance. Each panel represents the average stance time (time spent on 1 foot) per week while walking (vertical axis) over the entire study duration (horizontal axis). The rows of the panels represent long (top), medium (middle), and short (bottom) bouts, the columns of the panels represent either the full data set (left), the same data set down-sampled to only 6 continuous days of wear time per month (right), and to 5 2-month periods of wear time. The red data set is for the left foot and the blue-green data set for the right foot (affected side). See the legend of Figure 2 for details on the annotation. The level of compliance observed in this case study is exceptional, and we estimate that 6 continuous days per month, with $6 \mathrm{~h}$ per day (right panel), is sufficient to capture the major observations seen in the full data set (left panel), while a similar total wear time grouped into 2-month blocks is not.

\begin{tabular}{l}
\hline Digit Biomark 2018;2:79-89 \\
\begin{tabular}{l|l}
\hline DOI: 10.1159/000490919 & $\begin{array}{l}\text { c) 2018 The Author(s). Published by S. Karger AG, Basel } \\
\text { www.karger.com/dib }\end{array}$
\end{tabular} \\
\hline
\end{tabular}

Mueller et al.: Continuous Monitoring of Patient Mobility for 18 Months Using Inertial Sensors following Traumatic Knee Injury: A Case Study

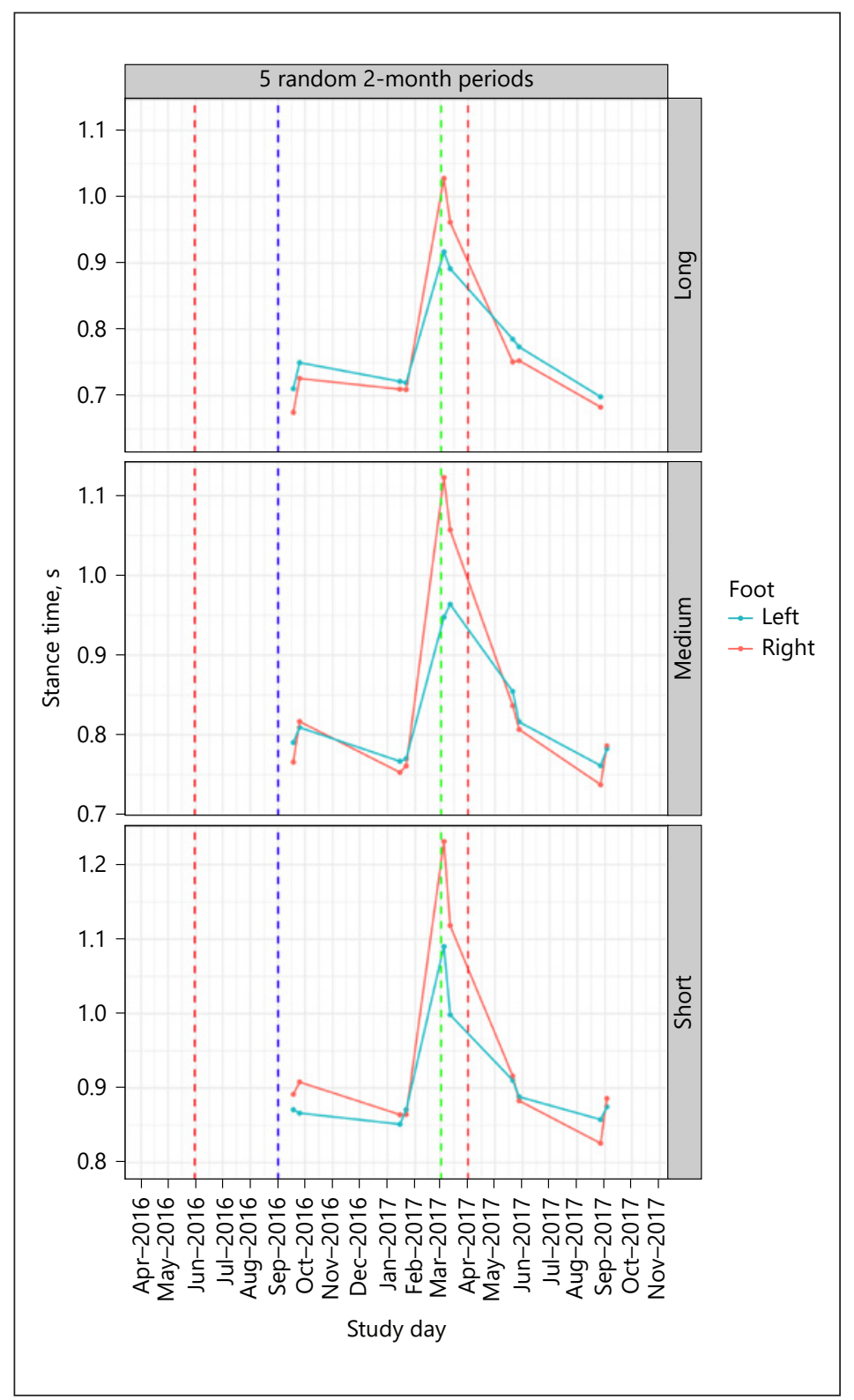

their relation to orthogonal measurements or data streams, for example muscle activation captured via electromyography, as sometimes done for osteoarthritis patients $[16,17]$. It should also be recognized that gait is not uniform between patients nor over time, particularly during injury, recovery, or intervention. Validation must therefore cater for this diversity through using a sufficiently heterogeneous cohort to characterize the limitations of any novel algorithm.

Despite the value described here, one limitation of accelerometry is that it does not directly capture contextual annotation (i.e., environmental conditions or other factors that would influence, or aid the interpretation of, captured data), reducing our ability to make meaningful comparisons over large time periods. In this case study, we group bouts of walking by length to allow comparison of similar periods of walking over time; however, it is likely that further annotation would help manage variation due to environmental factors and allow 
Mueller et al.: Continuous Monitoring of Patient Mobility for 18 Months Using Inertial Sensors following Traumatic Knee Injury: A Case Study

a more sensitive interpretation of the patterns observed. Precedence for such approaches exists, with studies already using mobile technologies to trigger or annotate inertial sensor and patient reported outcome data capture using environmental cues [18, 19].

This case study is a demonstration of the added value for approaches aiming at the digital continuous monitoring of patient mobility. We make our data available with the intention that other groups will identify further insights (e.g., other aspects of mobility beyond steps and gait speed including stair climbing or turning [20]), apply these methods to other indications (e.g., neurological conditions such as Parkinson's disease [5]) or use the data to develop device-agnostic code and algorithms [21]. This should enable application of these methods to the rapidly evolving technology landscape [6].

\section{Acknowledgements}

The authors would like to acknowledge the support, critical review, and assistance of Sarah Hemsley, Lorcan Walsh, Gerhard Aigner, Christian Lederer, Sophie Lemire-Brachat, Jason Laramie, Ronenn Roubenoff, and Jörg Goldhahn.

\section{Statement of Ethics}

Written informed consent was obtained from the participant for the collection and use of the data, and a local ethics committee waiver was granted for the collection and publication of the data.

\section{Disclosure Statement}

Ieuan Clay and Martin Daumer are members of the editorial board for Karger Digital Biomarkers. Martin Daumer is Scientific Director of SLCMSR e.V. and CEO of Trium Analysis Online $\mathrm{GmbH}$, makers of actibelt.

\section{Funding Sources}

The authors have no external funding sources to disclose.

\section{Author Contributions}

A.M., H.H., N.H., M.S., and I.C. contributed to the conception and design of data collection; A.M., T.N., N.H., M.D., and I.C. contributed to data acquisition; A.M., H.H., T.N., and I.C. performed the data analysis; interpretation of the data was performed by A.M., H.H., N.H., M.S., and I.C.; A.M., H.H., N.H., M.D., and I.C. drafted and revised the manuscript. 


\begin{tabular}{|c|c|}
\hline Digit Biomark 2018;2:79-8 & \\
\hline DOI: 10.1159/000490919 & $\begin{array}{l}\text { (c) } 2018 \text { The Author(s). Published by S. Karger AG, Basel } \\
\text { www.karger.com/dib }\end{array}$ \\
\hline
\end{tabular}

Mueller et al.: Continuous Monitoring of Patient Mobility for 18 Months Using Inertial Sensors following Traumatic Knee Injury: A Case Study

\section{References}

1 Giggins 0, Sweeney KT, Caulfield B: The use of inertial sensors for the classification of rehabilitation exercises. Conf Proc IEEE Eng Med Biol Soc 2014;2014:2965-2968.

2 Giggins OM, Sweeney KT, Caulfield B: Rehabilitation exercise assessment using inertial sensors: a crosssectional analytical study. J Neuroeng Rehabil 2014;11:158.

3 Kvedar JC, Fogel AL: mHealth advances clinical research, bit by bit. Nat Biotechnol 2017;35:337-339.

4 Prahm KP, Witting N, Vissing J: Decreased variability of the 6-minute walk test by heart rate correction in patients with neuromuscular disease. PLoS One 2014;9:e114273.

5 Bot BM, et al: The mPower study, Parkinson disease mobile data collected using ResearchKit. Sci Data 2016; 3:160011.

6 Clay I: Impact of digital technologies on novel endpoint capture in clinical trials. Clin Pharmacol Ther 2017; 102:912-913.

7 http://www.actibelt.com/.

8 Godfrey A, et al: Instrumenting gait with an accelerometer: a system and algorithm examination. Med Eng Phys 2015;37:400-407.

9 Motl RW, et al: Accuracy of the actibelt $\left({ }^{\circledR}\right)$ accelerometer for measuring walking speed in a controlled environment among persons with multiple sclerosis. Gait Posture 2012;35:192-196.

10 Schimpl M, et al: Association between walking speed and age in healthy, free-living individuals using mobile accelerometry - a cross-sectional study. PLoS One 2011;6:e23299.

11 Ithurburn MP, et al: Young athletes after ACL reconstruction with quadriceps strength asymmetry at the time of return-to-sport demonstrate decreased knee function 1 year later. Knee Surg Sports Traumatol Arthrosc 2018;26:426-433.

12 Reginster JL, et al: Guidelines for the conduct of pharmacological clinical trials in hand osteoarthritis: Consensus of a Working Group of the European Society on Clinical and Economic Aspects of Osteoporosis, Osteoarthritis and Musculoskeletal Diseases (ESCEO). Semin Arthritis Rheum 2017, Epub ahead of print.

13 Lynch $\mathrm{AD}$, et al: Current concepts and controversies in rehabilitation after surgery for multiple ligament knee injury. Curr Rev Musculoskelet Med 2017;10:328-345.

14 Archer KR, et al: Gait symmetry and walking speed analysis following lower-extremity trauma. Phys Ther 2006;86:1630-1640.

15 Hadizadeh M, et al: Assessment of gait symmetry improvements in national athletes after anterior cruciate ligament reconstruction during rehabilitation. Int J Sports Med 2016;37:997-1002.

16 Liikavainio T, et al: Gait and muscle activation changes in men with knee osteoarthritis. Knee 2010;17:69-76.

17 Lyytinen T, et al: Repeatability of knee impulsive loading measurements with skin-mounted accelerometers and lower limb surface electromyographic recordings during gait in knee osteoarthritic and asymptomatic individuals. J Musculoskelet Neuronal Interact 2016;16:63-74.

18 Reichert M, et al: Within-subject associations between mood dimensions and non-exercise activity: an ambulatory assessment approach using repeated real-time and objective data. Front Psychol 2016;7:918.

19 Tornros T, et al: A comparison of temporal and location-based sampling strategies for global positioning system-triggered electronic diaries. Geospat Health 2016;11:473.

20 Mancini $\mathrm{M}$, et al: Continuous monitoring of turning mobility and its association to falls and cognitive function: a pilot study. J Gerontol A Biol Sci Med Sci 2016;71:1102-1108.

21 Gilbert BK, et al: A measurement-quality body-worn sensor-agnostic physiological monitor for biomedical applications. Am J Biomed Eng 2015;5:34-66. 